Canadian University Music Review

Canadian University Music Review

Revue de musique des universités canadiennes

\title{
L’arbre mangé : Variations sur la chèvre qui sonne (Calabre, Italie)
}

\section{Bernard Lortat-Jacob}

Numéro 5, 1984

URI : https://id.erudit.org/iderudit/1013942ar

DOI : https://doi.org/10.7202/1013942ar

Aller au sommaire du numéro

\section{Éditeur(s)}

Canadian University Music Society / Société de musique des universités canadiennes

\section{ISSN}

0710-0353 (imprimé)

2291-2436 (numérique)

Découvrir la revue

Citer cet article

Lortat-Jacob, B. (1984). L’arbre mangé : Variations sur la chèvre qui sonne (Calabre, Italie). Canadian University Music Review / Revue de musique des universités canadiennes, (5), 242-268. https://doi.org/10.7202/1013942ar

All Rights Reserved ( C Canadian University Music Society / Société de musique des universités canadiennes, 1984
Ce document est protégé par la loi sur le droit d'auteur. L'utilisation des services d'Érudit (y compris la reproduction) est assujettie à sa politique d'utilisation que vous pouvez consulter en ligne.

https://apropos.erudit.org/fr/usagers/politique-dutilisation/ 


\title{
L'ARBRE MANGÉ : VARIATIONS SUR LA CHÈVRE QUI SONNE (Calabre, Italie)
}

\author{
Bernard Lortat-Jacob
}

L'impression qui prévaut à l'écoute de la musique d'Italie du Sud - et notamment de celle jouée sur les très belles cornemuses polyphoniques munies de deux tuyaux mélodiques et de deux ou trois bourdons, est celle d'une continuité sonore constamment altérée par un jeu de fines variations.

Ce jeu varié, ou microvarié, pour utiliser une expression de Carlo Crivelli (1979) constitue la base d'un système original qui, s'il a donné lieu à quelques considérations générales, n'a jamais fait l'objet d'une description détaillée ${ }^{1}$.

L'étude qu'on va lire prétend remédier à ce manque. À une exception près, elle s'appuie sur des documents déjà publiés. Il s'agit d'un travail de seconde main ayant pour base documentaire le disque Calabria publié à Rome par la Fonit Cetra qui comprend - outre de forts beaux enregistrements qui, du point de vue qui nous intéresse, présentent les cas les plus typiques une importante notice par Roberta Tucci et des notations musicales particulièrement complètes réalisées par Carlo Crivelli. En plus de cette publication majeure, d'autres exemples ont été pris en considération (cf. la discographie où les exemples sont numérotés de 1 à 4).

En Calabre, la cornemuse (zampogna, ciaramedda, ciarameja, ciaramelle, kärramunxia) est dite aussi capra che suona: la "chève qui joue et sonne " - le verbe suonare impliquant en italien ces deux notions. Dans ce jeu sonore, la mélodie variée est systématiquement distribuée en deux parties complémentaires correspondant à l'utilisation de deux tuyaux mélodiques joués simultanément qui sont insérés dans un bloc de bois (zippi, ou testale) ayant une fonction de distributeur d'air. Quelle 
qu'en soit la variété, ce jeu mélodique semble cependant mal se départir du jeu continu des bourdons constamment alimentés par une grosse réserve d'air; au point que les parties mélodiques ne prennent, par rapport à ces bourdons, qu'une faible autonomie et que la variation apparaît comme un jeu d'altération relativement timide par rapport à cette continuité.

Ce caractère est sensible dans les chants alla ciaramejara (à la cornemuse) dont les longues phrases s'articulent par paliers successifs en mouvements descendants se terminant sur le premier degré (degré bourdon). Il l'est également dans toutes les formes instrumentales, en particulier dans la tarantella.

Cette impression de continuité sonore ne trouve cependant pas son équivalent graphique dans les transcriptions de Carlo Crivelli que nous prendrons en exemple (tarantella, référ. disque 1 , face A, plage 2 et notation correspondante dans la figure 1). Non que ces transcriptions soient mauvaises - elles sont au contraire d'une finesse remarquable, même si, comme on verra, elles font appel à des notions solfégiques qui ne sont pas entièrement conformes au système qu'elles prétendent figurer - mais parce que le matériel thématique y est présenté selon des principes qui, selon nous, rendent mal compte des modalités de structuration de cette musique. Nous y reviendrons.

Dans le cas de la tarantella, cette structuration passe par une indispensable prise en considération de la danse. En effet, la tarantella qui, comme on sait, occupe une très grande place dans le répertoire d'Italie du Sud, est fondamentalement une musique de danse. Ainsi que l'écrit Giannattasio dans une courte étude non publiée (1979), le terme tarantella, dans toute l'Italie du Sud, désigne la danse en général ${ }^{2}$ et, de fait, la vie de la tarantella est entretenue par une pratique encore active de la danse - vie à laquelle tous les instruments, cornemuse bien sûr, mais aussi guitare "française » et guitare battente, accordéon diatonique, flûte double et, dans quelques cas isolés, viéle semblable en tout point à la lyra grecque, prêtent chacun pour leur part leur concours.

Cette danse, quelles que soient les variantes chorégraphiques observées, repose sur une alternance régulière de deux appuis portés alternativement et symétriquement sur les jambes gauche et droite et entre lesquels s'intercale éventuellement un saut plus ou moins important.

Un des traits fondamentaux de la forme musicale tarantella réside dans le fait que cette alternance est prise en compte par 
TARANTELLA

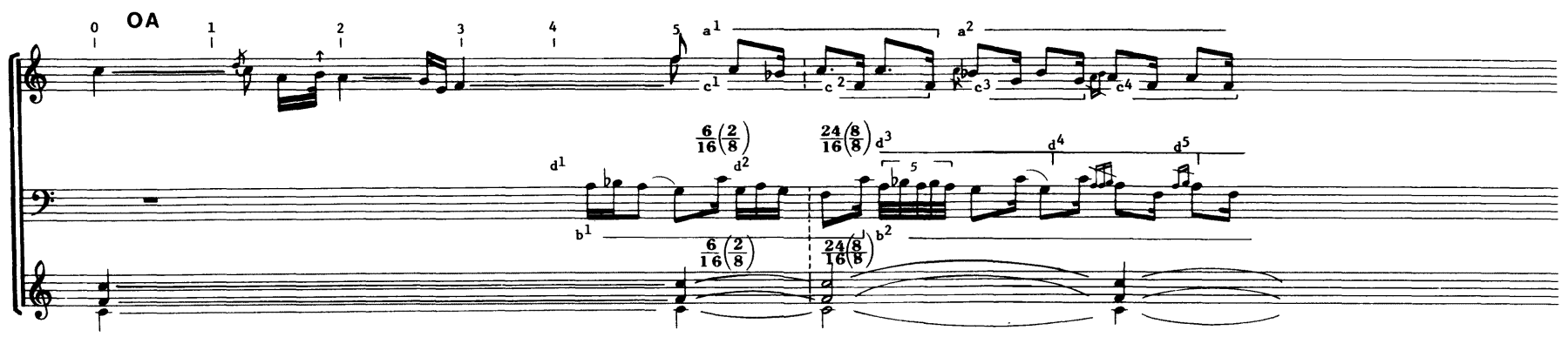

(1)

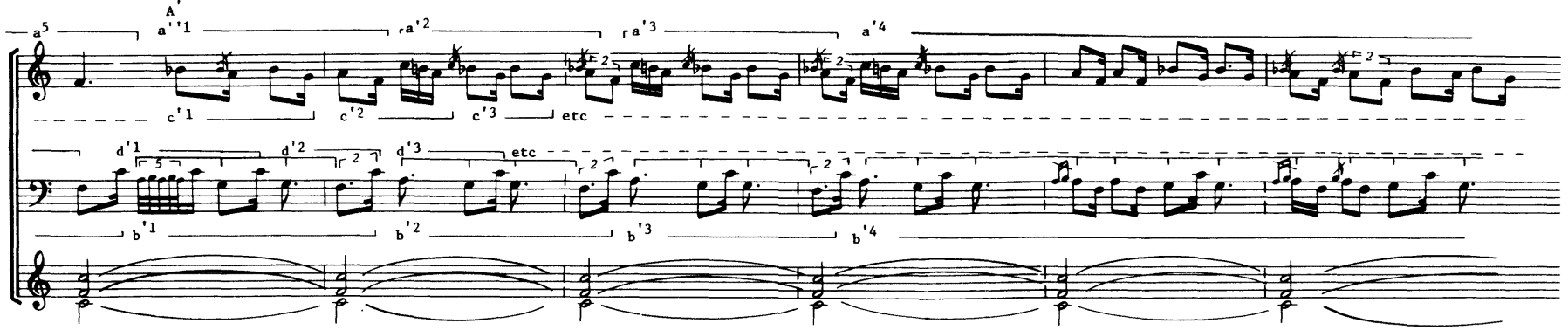





Figure 1

Tarantella, référence disque 1 , face $A$, plage 2 ; transcription de Carlo Crivelli, dans la notice de ce même disque, page 16. 
les instruments mélodiques : alternance d'accords de premier et cinquième degré pour la guitare; alternance de profils mélodiques se situant dans deux zones tonales opposées pour la cornemuse.

Dans l'exemple qui nous servira de référence (1, A-2), cette alternance régulière est visible; prenant dans la transcription qu'en donne Crivelli la demi-mesure équivalant à une noire pointée, on se rend compte que fa-premier degré, et sol-deuxième degré apparaissent en distribution complémentaire :

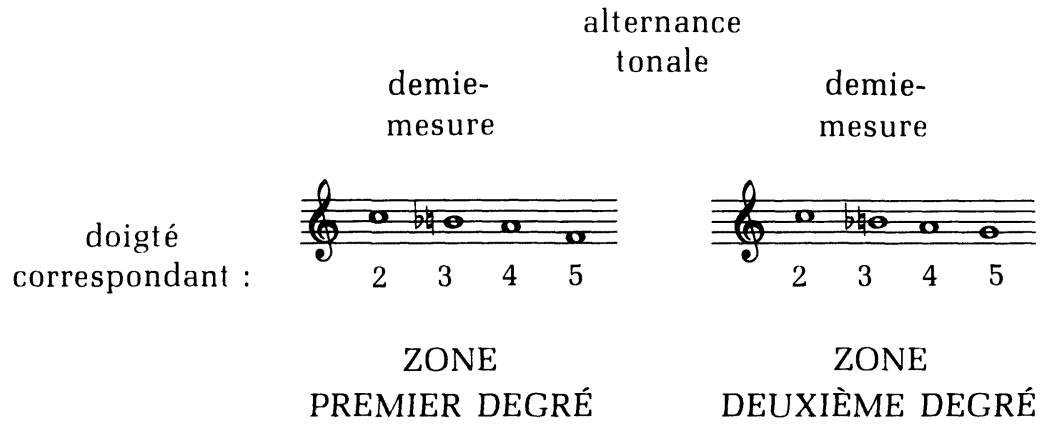

(N.B. : si est normalement bémolisé. Il est plus proche du bécarre à l'intérieur d'un mouvement ornemental descendant.)

Dans la danse, la zone tonale 1 s'articule avec l'appui sur une jambe; la zone tonale 2 avec l'appui sur l'autre jambe.

L'énoncé musical se structure donc en deux séries complémentaires, chaque série se voyant à tour de rôle sollicitée en un jeu de mesures régulières. Cette articulation est donnée dans la figure 2; les zones de premier degré apparaissent en grisé; celles de deuxième degré sont laissées en blanc. En outre, et pour faciliter l'analyse, après une courte période transitoire, les mesures ont été divisées en deux et numérotées respectivement à 1 à $n$ et de 1 ' à n'. Une hypothèse dès lors s'impose : la construction de l'énoncé ne repose pas sur l'articulation 1, 1', 2, 2', 3, 3', mais sur deux ensembles alternés 1, 2, 3 d'une part ; 1', 2', 3' d'autre part. Il reste que ces relations de dépendance du matériel thématique sont encore partiellement obscures; ceci du fait de la linéarité de l'écriture qui, censée retracer celle du discours musical, occulte les relations verticales.

Cependant, désirant proposer une mise en série des différents ensembles thématiques, un problème se pose. Pour 

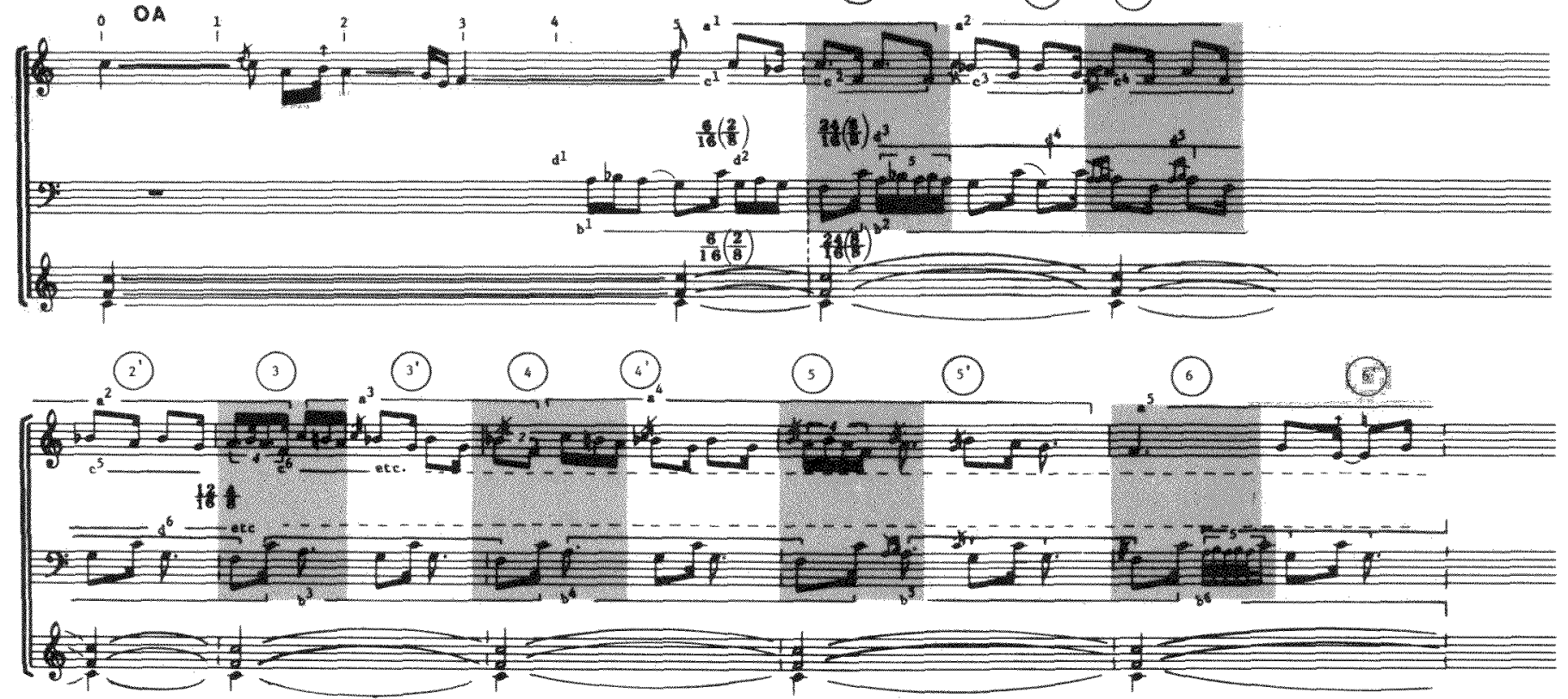

(1)

$\therefore 1$

(ㄱ) ()

(). ()

(iD) (10)

(11) (시)

(12)

(ii)

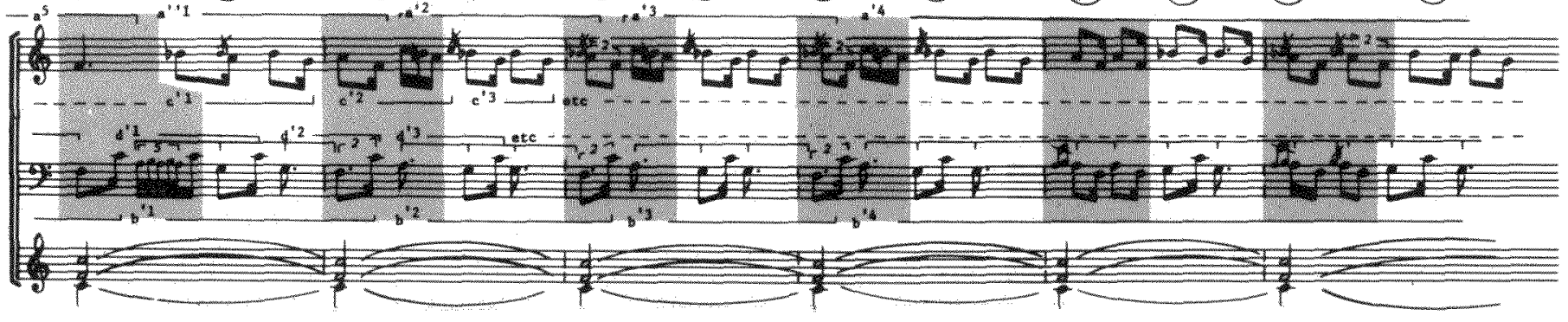


(13) (3)

(13) (10)

(14) 140

(15)

(15)

(16)

(26)

(17)

(17).

(13)

1." (18) 1912

$+\sqrt{1+2}$

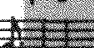
$12-2$

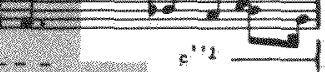

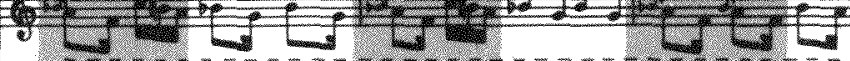

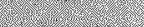

-

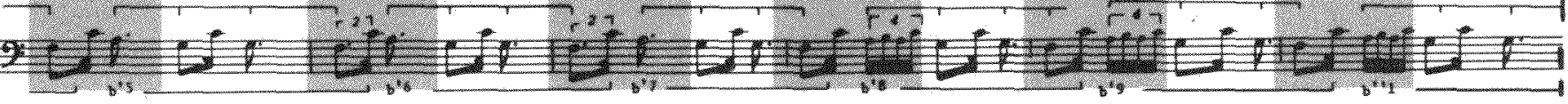

$4 \mathrm{C}=\mathrm{L}=\mathrm{C}$
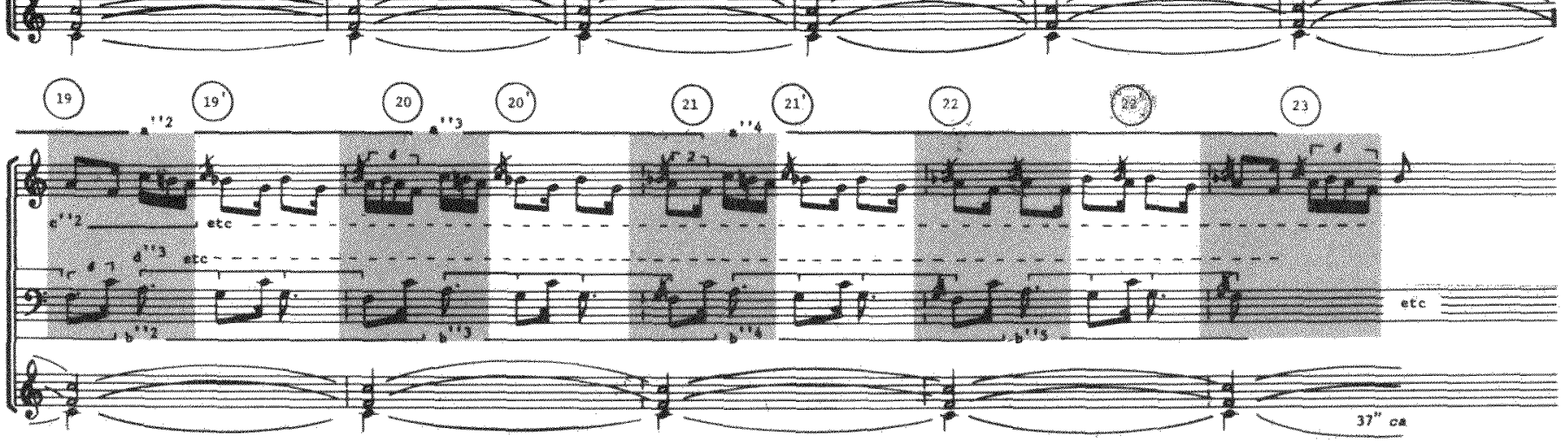

Figure 2

Tarantella, référence disque 1 , face $A$, plage 2.

Les zones grisées et non grisées, ajoutées sur la transcription de

Carlo Crivelli, renvoient aux zones tonales 1) et 2). 
rendre compte de la réalité paradigmatique, la notation synoptique effectuée selon les principes désormais classiques de Brailoiu, et visant à mettre en colonne les éléments récurrents en ne signalant que les variations, est bien connu de tous les ethnomusicologues. Quelqu'en soit l'intérêt ${ }^{3}$, cette méthode considère au moins implicitement comme acquis un certain nombre de présupposés sur les processus mis en ouvre dans l'exécution d'une musique : une transcription synoptique postule l'existence d'une unité de référence à laquelle sont rapportées un certain nombre de variantes; le paradigme étant constitué par la totalité des variantes dûment mises en colonne. Selon ces principes, la première unité (formant souvent strophe) se voit nécessairement bénéficier d'un statut particulier. Elle est en quelque sorte thème des variations à venir. "Tête de paradigme ", pour reprendre l'expression de J.-J. Nattiez (1975:264), elle a, du fait de sa position première (et sauf, bien entendu, indication contraire de l'analyste), une fonction structurante privilégiée.

Les musiques à thème et variations - fort fréquentes il est vrai dans les répertoires européens - sont celles qui se prêtent le mieux à une transcription synoptique traditionnelle. Bon nombre de musiques cependant, par rapport à cette méthode opérant sur une classe unique où l'incipit formant thème occupe une place privilégiée, se trouvent en quelque sorte en porte-à-faux. Ainsi celle de launeddas, où il serait erroné de privilégier un thème puisque le système en fait intervenir plusieurs en concurrence et que le matériau musical s'élabore non à partir d'une entité fixe où se trouvent cristallisées des notes en un ordre déterminé, mais en quelque sorte sur lui-même, à l'intérieur de sa propre continuité. Dans ce cas, la structuration s'effectue non à partir de classes constituées, mais à partir d'un principe dynamique (renouvellement/répétition) où chaque formule s'affirme non par rapport à un point de départ, mais par rapport au matériel précédemment exposé (cf. Lortat-Jacob 1981).

Le cas qui présentement nous occupe mérite, on va le voir, quelques considérations particulières. Un premier inventaire des composantes nous mettra sur la voie. D'emblée, la double structuration de l'espace tonal nous suggère deux mises en série ${ }^{4}$. Double mise en série qui se quadruple dès lors qu'on prend en considération le fait que la demi-mesure se compose elle-même de deux parties qui ne sont équivalentes sur le plan fonctionnel : la deuxième se différencie de la première en ce sens qu'elle peut avoir une valeur conclusive ou suspensive; une tarantella ne se 
clôt jamais sur a (ni d'ailleurs sur a'), mais sur b : exceptionnellement, elle peut se conclure sur b'. Ces données sont figurées par le graphe suivant :

Bourdon

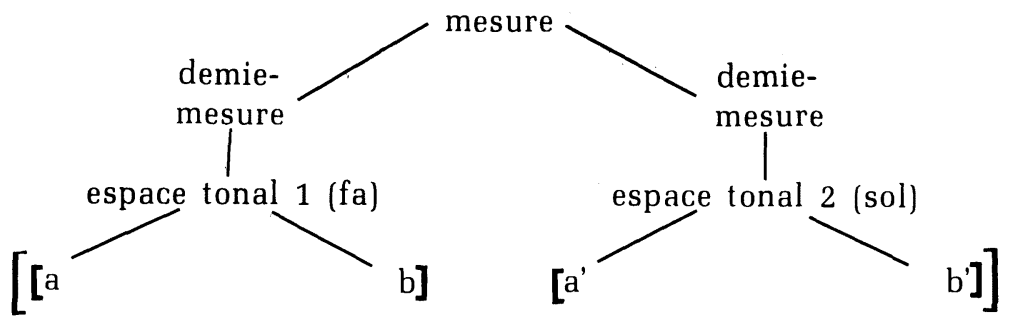

Communes à ces deux espaces tonals - deux composantes constituant, chacune pour leur part, des niveaux de structuration fondamentaux - 1) un bourdon continu, qui constitue une référence tonale constante; 2) une pulsation métrique distribuée en deux parties mélodiques et où s'affirment des relations constantes d'alternance longue-brève. Relations binaires dans le cas qui nous occupe (mais qui dans d'autres exemples, peuvent être ternaires) et que les conventions solfégiques adoptées par Crivelli dans sa transcription traduisent par $\stackrel{\tau}{7}$

Les espaces tonals de base et la pulsation métrique composite élémentaire - en d'autres termes, nos deux premiers niveaux sont représentés dans la figure 3 . Au point où nous en sommes de l'analyse, l'absence d'une référence à tout monnayage ternaire nous dispense de la mention de duolet.

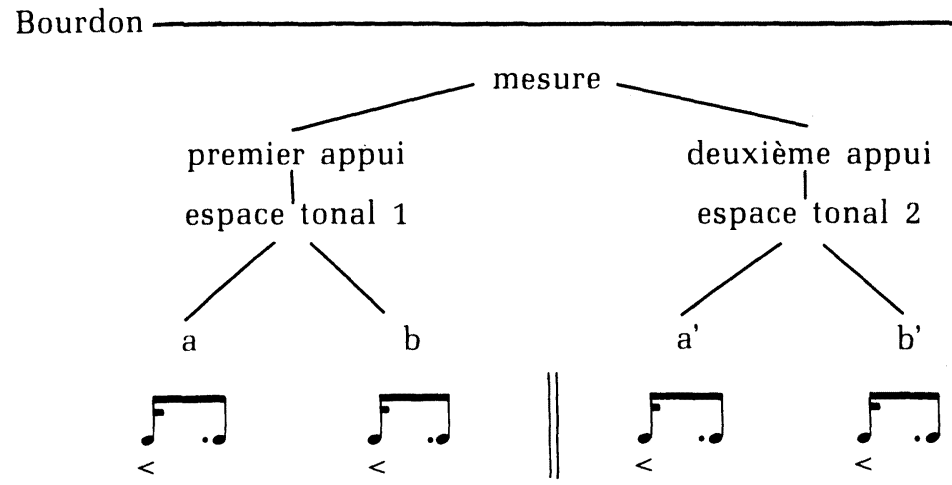

Figure 3 
Clarifions ce que l'écriture laisse en grande partie dans l'ombre et qui concerne les modalités d'accentuation bien caractéristiques, inverses des conventions ou habitudes classiques qui, le plus souvent, associent longueur et accentuation. Ici au contraire, la brève est très nettement accentuée. D'où le recours au signe $>$ qu'il ne convient pas de considérer comme un accent rythmique accidentel, mais comme un élément constitutif de la structure métrique de base.

Cette structuration métrico-tonale peut être obtenue sur tout instrument susceptible de générer deux sons ${ }^{5}$. Et à partir d'elle la danse est possible (cf. par exemple la tarantella "minimale », figurant dans le disque 2, face A, plage 4).

Les possibilités conclusives ou suspensives déjà mentionnées opérant sur b (plus rarement sur b') conduisent à la transformation des deux ensembles croche pointée-double en leur équivalent en longue (à savoir : blanche).

Dans le cas qui nous occupe, - mais on verra comment ces faits sont porteurs de généralité - , cette structure métricotonale de base, toujours explicite, accepte de façon systématique un jumelage avec une structure qui la contredit sur le plan métrique, mais non sur le plan tonal (c'est-à-dire que l'alternance tonale est en tout état de cause respectée) : chaque cellule peut être le lieu d'un monnayage ternaire qui, en un niveau de structuration distinct $\left(3^{\mathrm{c}}\right.$ niveau) conduit à la constitution d'ensembles simples ou composites; ainsi :

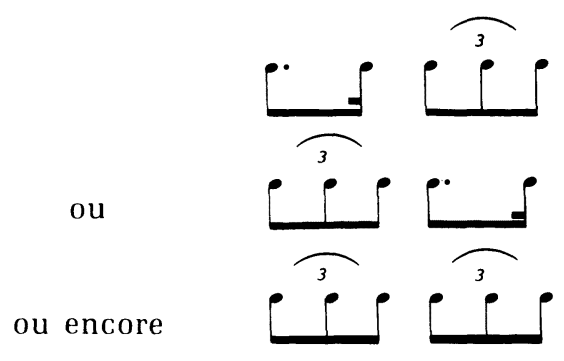

Figure 4

Figurations ternaires de niveau 3

La ligne de force fondamentale de la tarantella se dégage de la juxtaposition simultanée ou différée dans la chaîne de ces sous-ensembles binaires et ternaires. L'imprévisibilité de leur apparition (pour l'auditeur tout au moins) autant que leur 
nature fondamentalement antagoniste confère à la danse un dynamisme métrico-rythmique très caractéristique.

Ces deux composantes qui, selon les styles d'exécution, agissent en alternance ou en concurrence directe, se voient chacune pour leur part, complétées par des réalités ornementales variées; le principe de cette ornementation consistant précisément à opérer un sous-monnayage des valeurs de base minimales (c'est-à-dire avec des valeurs plus brèves que la croche pour la structure binaire et la croche en triolet pour la structure ternaire).

Dans la musique instrumentale, jouée en particulier sur un tempo vif et par des instruments à vent, l'ornementation, tant pour des raisons mécaniques qu'acoustiques, procède par mouvement conjoint. Nous verrons selon quelles modalités un peu plus loin. Retenons cependant que les différentes expansions ornementales qui peuvent consister en une simple note ou en des ensembles formulaires un peu plus importants 1) s'intercalent entre les valeurs minimales (elles-mêmes de courte durée), et 2), sauf exception, sont en relations conjointes avec les éléments qu'elles ornent.

À ce point de l'analyse, une remarque critique s'impose. Elle concerne la transcription de la figure 1 qui jusqu'à présent nous a servi de base d'analyse, et procède directement de la difficulté que nous rencontrons à juger comme adéquates certaines figures ornementales relevées par Crivelli, et que nous rappelons dans la figure 5 .

À dire vrai, le luxe de détails donnés dans la transcription, et la complexité des figures solfégiques proposées semblent mal " coller " à l'image mentale que nous procure l'écoute réitérée de cette tarantella. La puissante dynamique binaire-ternaire, déjà relevée, qui pourtant, dans le présent exemple, s'exerce sans le secours d'instruments à percussion, semble mal s'accommoder de cette profusion de détail. Encore que ce ne soit pas tant cette profusion en tant que telle qui nous gêne, mais plutôt la difficulté que nous rencontrons à afférer une quelconque réalité à des figures de doubles-croches en quartolet, et a fortiori, de triples-croches en quintolet. Notre hypothèse est que le niveau ornemental ne constitue pas un niveau autonome; l'impression dominante étant que l'ornementation participe étroitement de la structuration de base binaire-ternaire. 


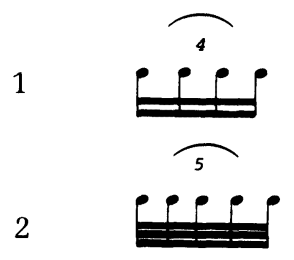

Figure 5

Figures ornementales selon Crivelli (cf. fig. 1)

Sans doute le niveau ornemental complique-t-il sensiblement cette structuration métrique ; mais, en tout état de cause, celle-ci reste perceptible. De fait, ce problème d'ornementation se clarifie considérablement dès lors qu'on le considère dans ses rapports avec la structure mélodico-tonale et avec la structure métrique de base :

- les notes ornées sont exclusivement la dans la zone tonale 1) et si dans la zone tonale 2) ; c'est-à-dire que l'ornementation n'opère que sur la tierce des deux degrés fondamentaux et qu'en outre, elle prend la forme d'une broderie supérieure qui introduit par mouvement conjoint la note ornée - broderie simple ou double sur les valeurs longues; broderie simple sur les valeurs brèves. Cas particulier : à l'intérieur de la cellule ternaire, il y a deux broderies simples lorsque celle-ci se compose de notes répétées.

Ces données sont récapitulées dans la figure 6 où les notes d'ornement sont barrées :

zone tonale 1

1

2

3
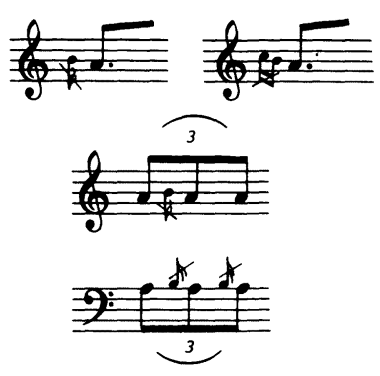

zone tonale 2

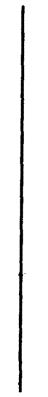

Figure 6

1. Ornementation sur valeur longue correspondant à un temps fort;

2. Ornementation sur valeur brève (temps faible): 3. Cas particulier de cellule avec notes mélodiques répétées. 
La notation 1 de la figure 6 est conforme aux conventions de la figure 1 (Crivelli). 2 et 3 de cette même figure doivent être substitués respectivement à 1 et 2 de la figure 5 .

Selon ces principes, l'ornementation, on le voit, apparaît comme une sophistication relativement simple de la structuration binaire (sur les valeurs longues) et ternaire (sur les brèves).

Avant de proposer une transcription sur ces nouvelles bases, une autre observation s'impose : la transcription de la figure 1 a pour référence une unité ternaire. Cette convention est malheureuse à partir du moment où l'on considère que l'unité fondamentale ayant une fonction déterminante pour la structure est l'unité binaire donnant à la danse une impulsion constante. Aussi, tant qu'à adopter une convention solfégique, nous préférerions partir de cette structuration binaire et trioliser ce qui doit l'être. Cette écriture n'est certes pas totalement satisfaisante puisque les conventions solfégiques qui la soustendent impliquent que le ternaire est en quelque sorte dérivé du binaire, ce qui, en tout état de cause, n'est pas conforme aux conceptions métriques en jeu dans la tarantella, où, comme nous l'avons écrit plus haut, binaire et ternaire apparaissent comme fondamentalement antagonistes ${ }^{6}$. Le ternaire relève d'un niveau propre (troisième niveau) et ne procède pas d'un monnayage d'une unité binaire déjà constituée. On rappellera que si binaire et ternaire constituent chacun pour leur part des niveaux autonomes, il n'en va pas de même pour l'ornementation qui ne constitue pas en soi un niveau distinct des deux autres.

Bien que l'écriture solfégique soit incapable de rendre compte de faits si importants, pour des raisons de pratique de lecture évidente, on s'en satisfera. Ultime précision : pour aider également la lecture, nous avons pris pour convention de transcrire les figures métriques de base avec les hampes vers le haut pour la zone tonale 1 , vers le bas pour la zone tonale 2. La figure 7 présente une transcription conforme à ces principes; la figure 8 reprend les éléments constitutifs du système : 


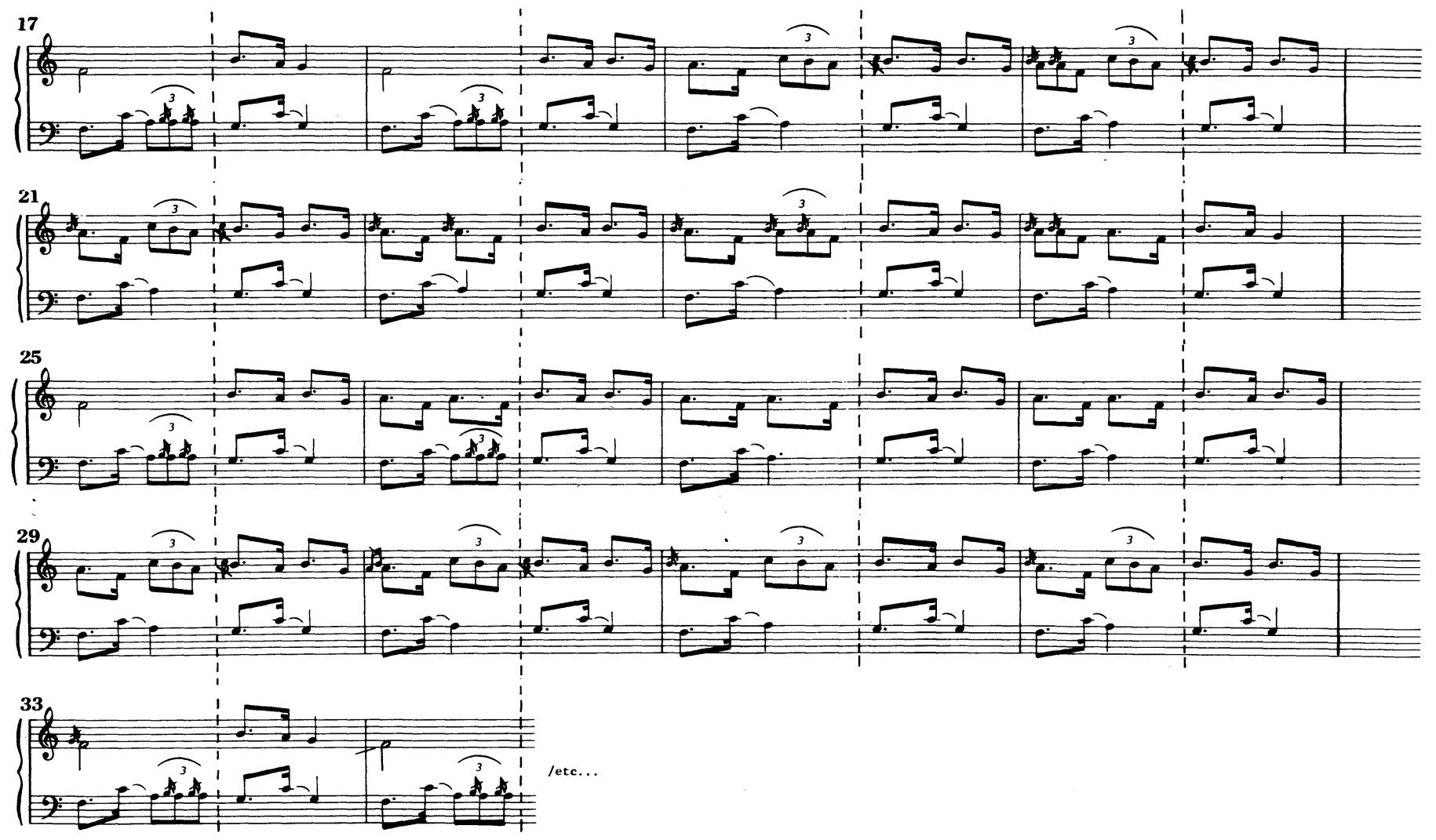

Figure 7 


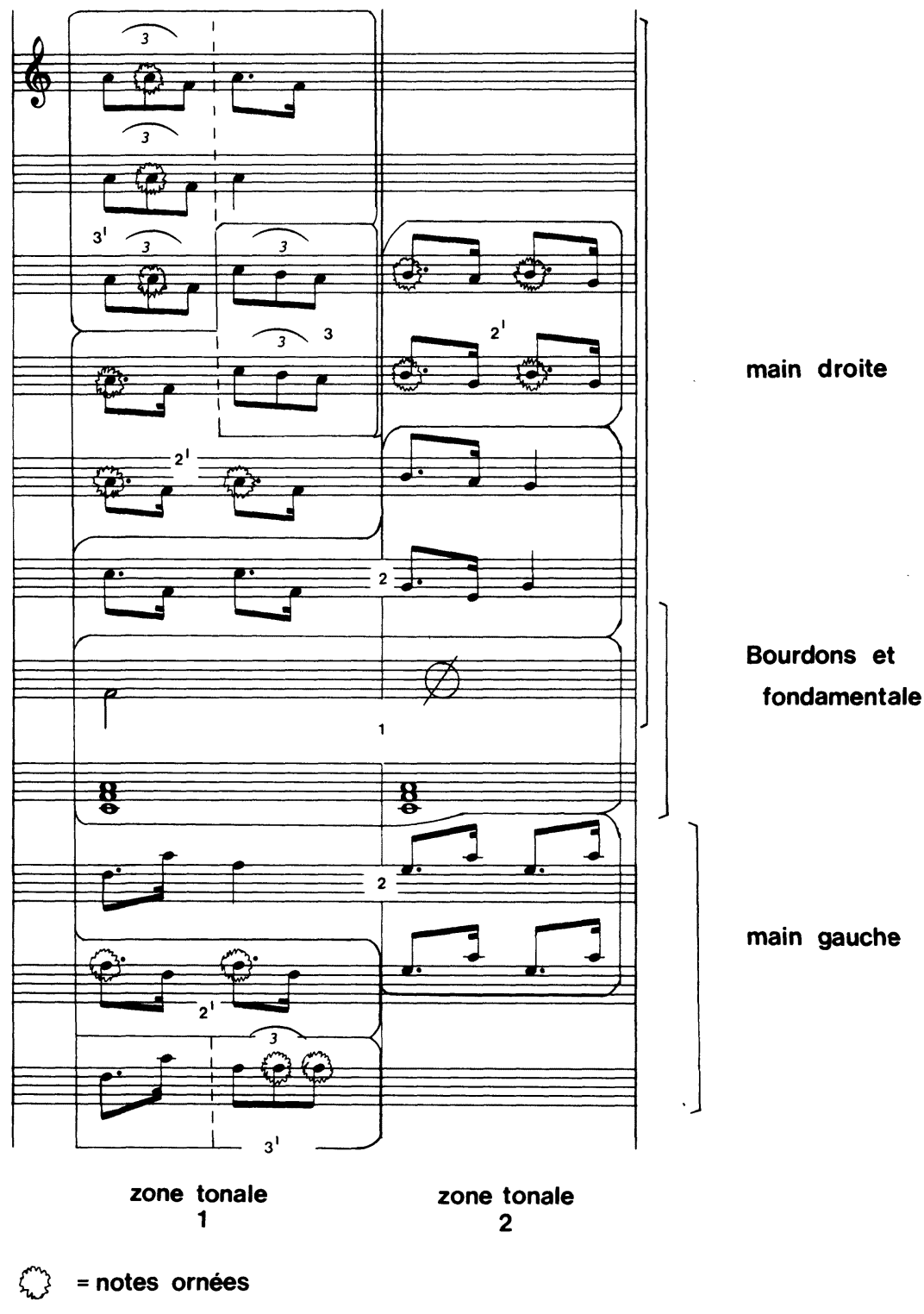


Comme on peut le voir sur ces deux figures présentant la totalité de notre matériel musical, et sur lesquelles nous reviendrons, la structuration mélodique obéit à des exigences de mouvements contraires main gauche/main droite (MG/MD). En terme de technique de jeu, lorsque MG travaille sur son chalumeau de haut en bas, MD travaille de bas en haut, et réciproquement. Par ce procédé, les notes doublées sont évitées en même temps que les mouvements parallèles et chaque zone tonale est exploitée dans toute son épaisseur. Les parties mélodiques, se croisant en des figures diverses, apparaissent dès lors comme des variations sur des accords parfaits qui s'accommodent d'interventions furtives de notes de passage en valeurs brèves (cf. fig. 9) :

zone tonale $1 \quad$ zone tonale 2

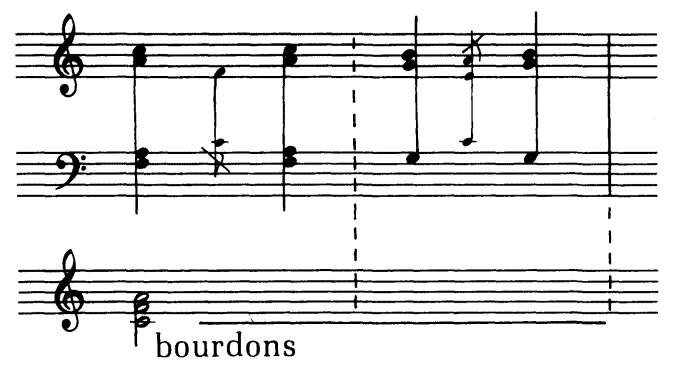

Figure 9

Le bourdon double et renforce l'harmonie de la zone tonale 1 et contraint toutes figurations mélodiques de zone tonale 2 à se présenter comme éléments d'une divergence conduisant à une résolution dans la zone tonale fondamentale.

La zone tonale 1, combinant des rapports de consonance est, comme nous le verrons un peu plus loin en détail, la plus riche en variations, et celle qui mobilise la plus grande inventivité musicale. La zone tonale 2 se cantonne en revanche dans un jeu de variations beaucoup plus réduit. La forme tout entière s'inscrit dans une continuité tonale que le jeu de variations vient altérer - très timidement en zone tonale 2 , un peu moins en zone tonale 1.

Ces variations sont recensées et ordonnées dans la figure 8 en fonction de leurs traits constitutifs et discriminatoires : 
zone tonale, mètre, ornementation. Elles forment des classes spécifiques disposées en miroir à partir d'un axe tonal central assurant la continuité harmonique.

La structuration s'opère de la façon suivante (nous utiliserons les abréviations MG, MD pour main gauche/main droite, et ZT $1 / \mathrm{ZT} 2$ pour zone tonale 1 et 2) :

- classe 1, Bourdons et fondamentale : fondamentale en valeur longue par MD exclusivement.

- classe 2, Composante métrique binaire élémentaire, sans ornementation : cellule élémentaire ( $\digamma$ ) excluant toute ornementation; attestée à la fois à MG et MD, en ZT 1 et ZT 2.

- classe 2', Composante métrique binaire, avec ornementation : cf. classe précédente mais avec restriction : apparaît en ZT 2 seulement à MD.

- classe 3, Composante métrique ternaire, sans ornementation : exclusivement en ZT 1 et par MD.

- classe 3', Composante métrique ternaire, avec ornementation : en ZT 1 et ZT 2; figurations combinées à l'intérieur de la mesure (cf. infra).

Ces classes sont sollicitées dans la chaîne selon certains principes qu'il nous faut maintenant considérer en nous référant au tableau ci-dessous qui représente synthétiquement l'exemple qui nous sert de référence :

\begin{tabular}{|c|c|c|c|c|c|c|c|c|}
\hline & ZT1 & ZT2 & ZT1 & $\mathrm{ZT} 2$ & ZT1 & $\mathrm{ZT} 2$ & ZT1 & ZT2 \\
\hline $\mathrm{MD}$ & 2 & 2 & $2^{\prime}$ & $2^{\prime}$ & $3^{\prime}$ & $2^{\prime}$ & 3 & $2^{\prime}$ \\
\hline $\mathrm{MG}$ & $3^{\prime}$ & 2 & $2^{\prime}$ & 2 & 2 & 2 & 2 & 2 \\
\hline $\mathrm{MD}$ & $3^{\prime}$ & $2^{\prime}$ & 1 & 2 & 1 & 2 & 3 & $2^{\prime}$ \\
\hline $\mathrm{MG}$ & 2 & 2 & $3^{\prime}$ & 2 & $3^{\prime}$ & 2 & 2 & 2 \\
\hline $\mathrm{MD}$ & 3 & $2^{\prime}$ & 3 & $2^{\prime}$ & 2 & 2 & $2^{\prime}$ & 2 \\
\hline $\mathrm{MG}$ & 2 & 2 & 2 & 2 & $2^{\prime}$ & 2 & $2^{\prime}$ & 2 \\
\hline $\mathrm{MD}$ & 3 & 2 & 3 & $2^{\prime}$ & 2 ' & 2 & 1 & 2 \\
\hline$\overline{\mathrm{MG}}$ & 2 & 2 & 2 & 2 & 2 & 2 & $3^{\prime}$ & 2 \\
\hline $\mathrm{MD}$ & 1 & 2 & 1 & 2 & 3 & $2^{\prime}$ & $3^{\prime}$ & $2^{\prime}$ \\
\hline $\mathrm{MG}$ & $3^{\prime}$ & 2 & $3^{\prime}$ & 2 & 2 & 2 & 2 & 2 \\
\hline $\mathrm{MD}$ & 3 & 2' & $2^{\prime}$ & 2 & $3^{\prime}$ & 2 & $3^{\prime}$ & 2 \\
\hline MG & 2 & 2 & 2 & 2 & 2 & 2 & 2 & 2 \\
\hline $\mathrm{MD}$ & 1 & 2 & 2 & 2 & 2 & 2 & 2 & 2 \\
\hline $\mathrm{MG}$ & $3^{\prime}$ & 2 & $3^{\prime}$ & 2 & 2 & 2 & 2 & 2 \\
\hline $\mathrm{MD}$ & 3 & 2 & 3 & 2 & 3 & 2 & 3 & 2 \\
\hline $\mathrm{MG}$ & 2 & 2 & 2 & 2 & 2 & 2 & 2 & 2 \\
\hline$\underline{\mathrm{MD}}$ & 1 & 2 & 1 & & & & & \\
\hline $\mathrm{MG}$ & $3^{\prime}$ & 2 & 3 & & & & & \\
\hline
\end{tabular}

mes. 1 à 4

5 à 9

10 à 14

15 à 18

19 à 22

23 à 26

27 à 30

31 à 34 
Les combinaisons attestées sont les suivantes :

$\begin{array}{llllllllll}\text { MD } & 2 & 2 & 3 & 3 & 1 & 2 & 2 & 2 & \text { pour la zone tonale } 1 \\ \text { MG } & 3 & 2 & 2 & 2 & 3 & 2 & 2 & 2 & \text { por }\end{array}$

$\begin{array}{llll}\text { MD } & 2 & 2 & \text { pour la zone tonale } 2\end{array}$

En fait, les combinaisons théoriquement possibles sont au nombre de 2 pour la ZT 2 (et de ce point de vue là, nombres théorique et pratique correspondent) et de quinze pour la ZT1; or huit seulement sont attestées qui, dans le tableau suivant, sont marquées d'un astérisque :

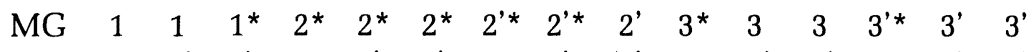

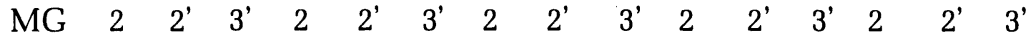

Le graphe suivant (fig. 10) met en évidence les relations MG/MD attestées pour chaque zone tonale :

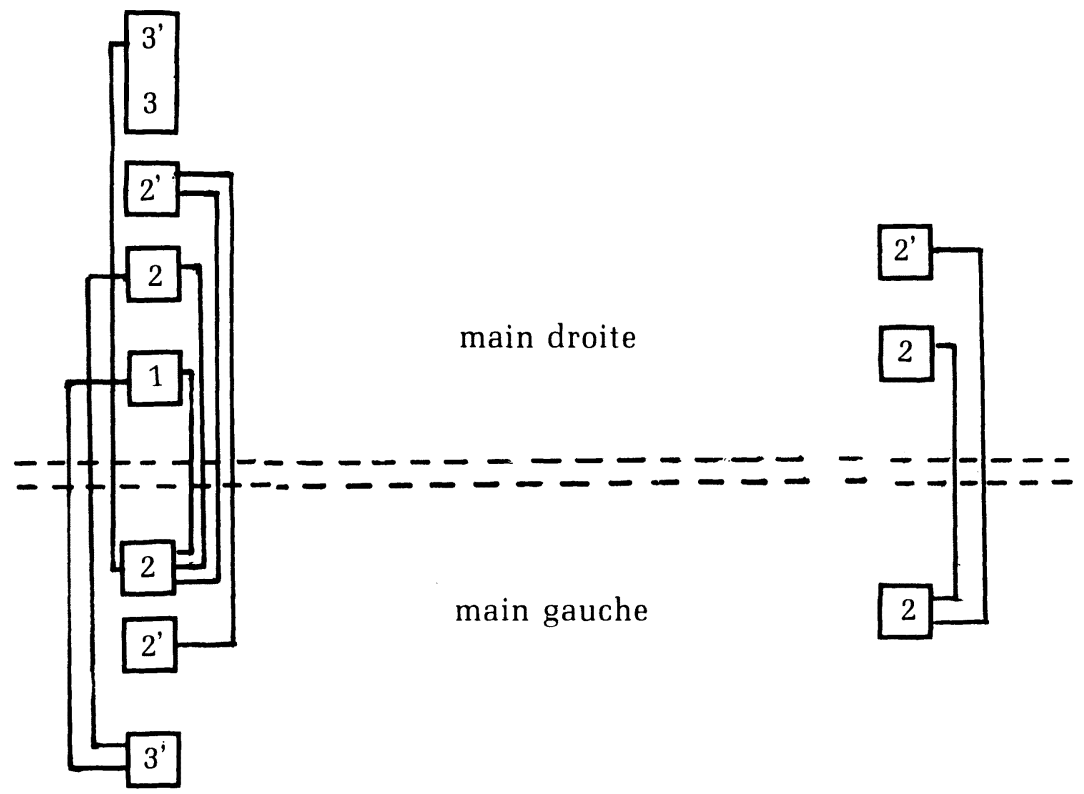

zone tonale 1

zone tonale 2

Figure 10

Articulations binaire ternaire $M G / M D$; à droite de chaque figure : relations impliquant binaire/binaire; à gauche : binaire/ternaire 
1) La classe 1 où, à la main droite exclusivement, s'inscrit la fondamentale en valeur longue, s'accommode soit d'une structure strictement binaire non ornée à la main gauche, soit d'une ornementation sur une base ternaire;

2) la classe 2, binaire non ornée, voyant en cela son rôle structurel fondamental considérablement renforcé, accepte toutes les possibilités combinatoires;

2') les ornementations binaires ne sont associées qu'à la classe binaire sollicitée par la main opposée; ornementations binaire et ternaire ne sont pas compatibles en jeu simultané ; 3 et $\left.3^{\prime}\right)$ les figurations ternaires, lorsqu'elles n'ornent pas la longue ternaire (cf. 1), fonctionnent sur une base binaire non ornée. Ceci exclut que les deux mains jouent en ternaire simultanément et renforce l'antagonisme binaire/ternaire dont on a dit qu'il imprimait à la tarantella son dynamisme fondamental. Le ternaire, dans le cas qui nous occupe, passe d'une main à l'autre dans un jeu de lignes alternées que vient constamment briser la structure permanente binaire.

Bourdons, contraintes harmoniques et tonales, composantes métriques binaire/ternaire, articulation $\mathrm{MG} / \mathrm{MD}$, jeu de variations et d'ornementations sont les données constitutives de la tarantella. La figure 11 rend compte de l'articulation de ces données. Sa lecture doit être complétée par un recours à la figure 8 recensant, sur des portées, les énoncés particuliers composant le terminal de la structure en arbre : 


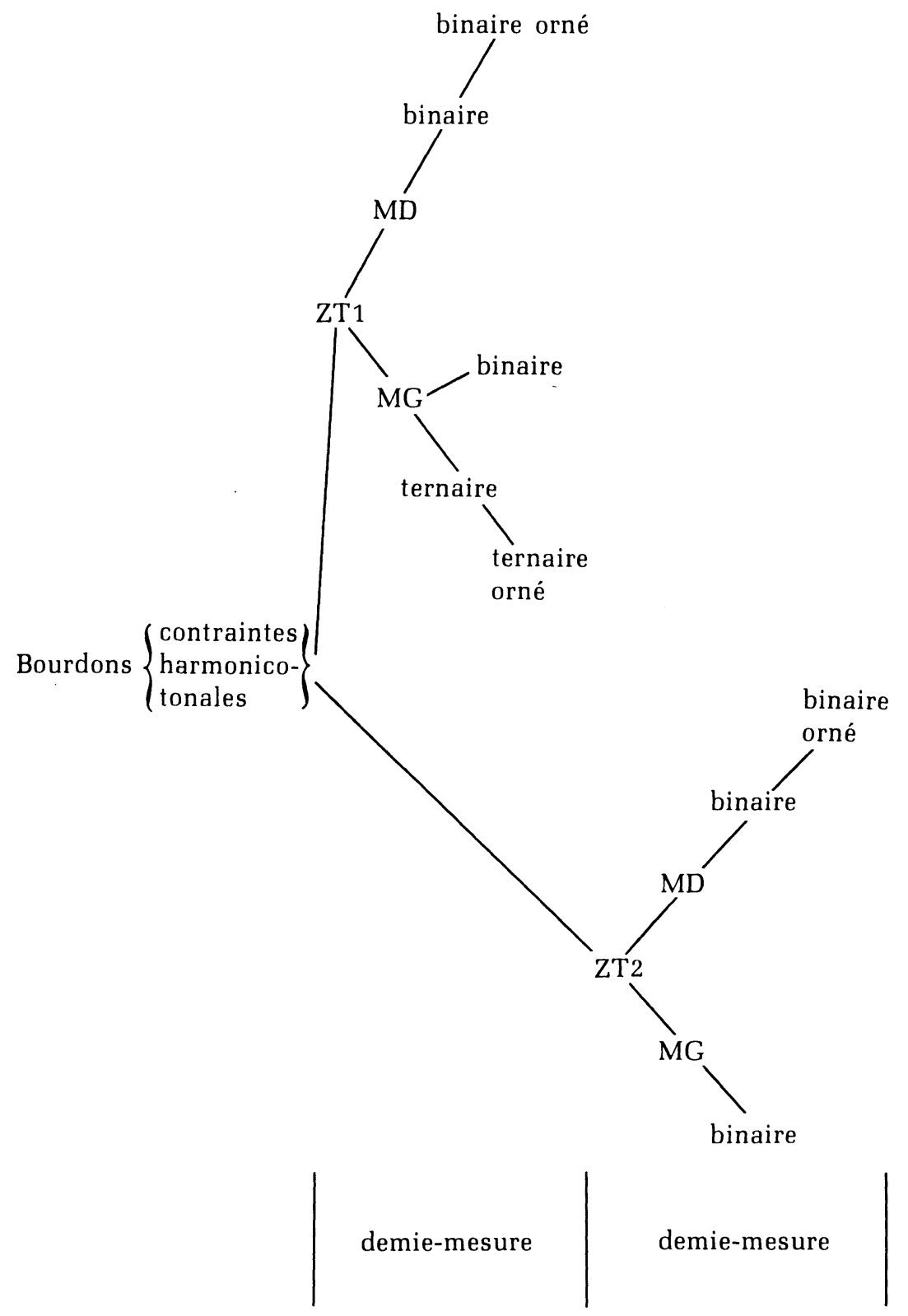

Figure 11

Composantes ordonnées de la tarantella. Les ramifications ont pour terminaison les classes recensées pour notre exemple (cf. fig. 8) 
La figure 11 ne prétend pas donner de la tarantella une description générative complète où l'arbre représenterait la tarantella et seulement elle. Elle propose un inventaire organisé des différentes sous-structures entrant dans la forme tarantella. Les composantes sont : bourdons, contraintes harmonico-tonales, ZT 1 et 2 se distribuant en parties MG/MD. À partir de cette base commune à toutes les tarantelle jouées à la cornemuse polyphonique s'ajoutent des possibilités variées d'alternance et de combinaisons binaire/ternaire/orné/non orné qui donnent d'importantes possibilités combinatoires de variations. Ces possibilités s'incarnent dans des énoncés formulaires en nombre limité qui, en fonction de leurs traits discriminants, forment des classes distinctes. La technique de variation revient à opérer des choix à l'intérieur de ces classes. Les modalités de combinaison de ces classes répondent à des principes déjà mentionnés et figurés dans le graphe de la figure 10. Le choix des énoncés formulaires à l'intérieur de ces classes obéit, quant à lui, au principe de la variation obligée. Alors que deux classes peuvent sans inconvénient être répétées deux fois de suite dans des combinaisons identiques, l'alternance des énoncés formulaires est plus strictement contrainte au principe de la variation, c'est-à-dire de la non répétition : les répétitions formulaires de ZT 1 à ZT 1 (occupant donc chaque première demi-mesure) sont limitées au maximum; celles de ZT 2 à ZT 2 (seconde demi-mesure), du fait que le nombre de formules possibles est moins élevé, échappe en partie à ce principe.

Précisons que le sens de la variation en général porte à la fois sur ZT 1 et ZT 2, mais en des termes qui ne sont pas équivalents. Dans l'exemple suivant (disque 1, face B, plage 6), nous avons deux micro-structures complémentaires : ZT 1, composé de do dièse, ré + mi comme note ornementale; ZT 2, composé de si, ré et do dièse en note de passage; l'ambiguïté binaire/ ternaire est diffuse durant toute la séquence (fig. 12) : 


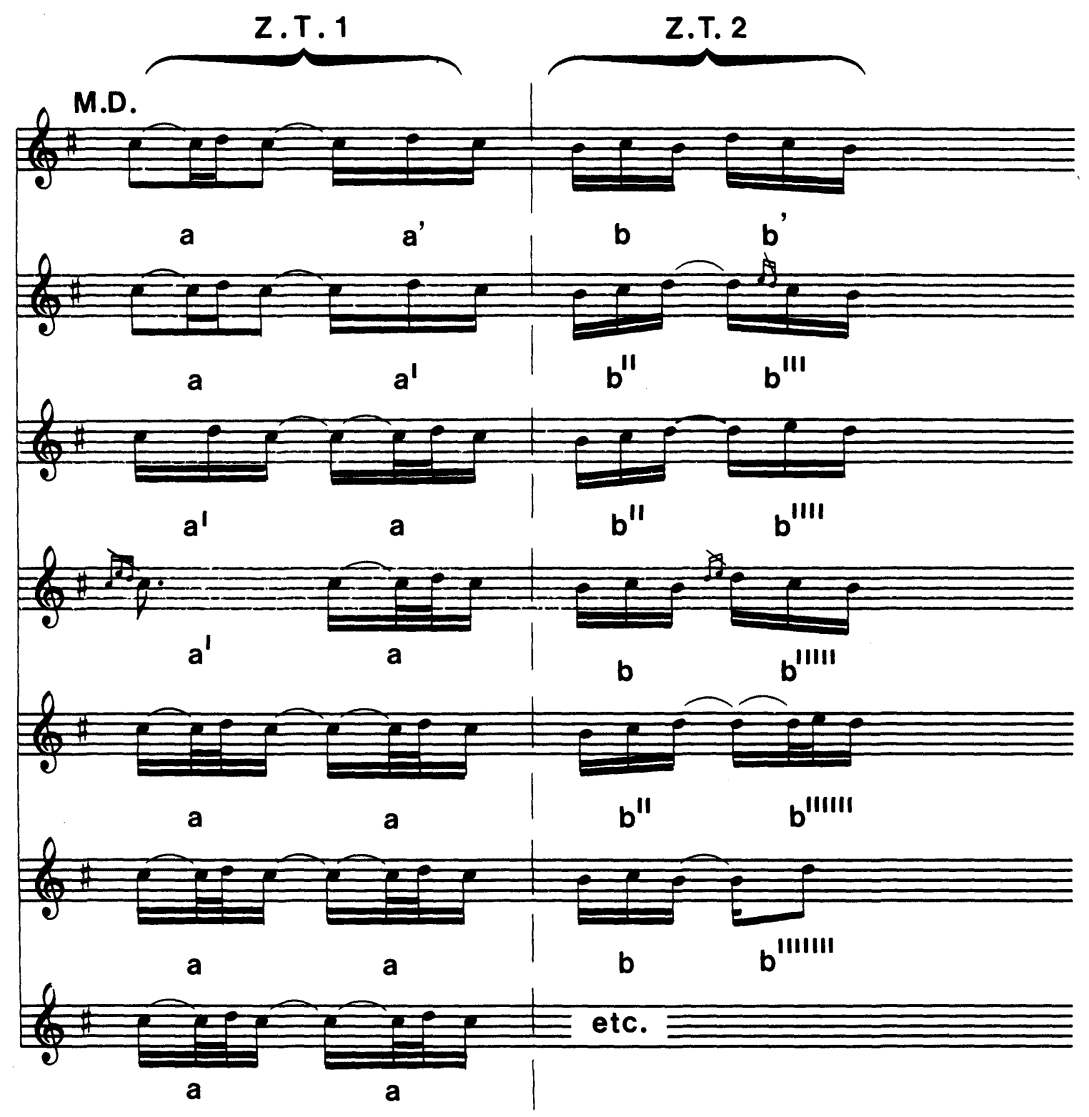

Figure 12

La structure en arbre a également l'avantage de nous faire comprendre - mais nous ne nous attarderons pas sur ce point qui mériterait à lui seul une étude particulière - comment, par exemple l'accordéon diatonique a pu prendre à son compte la tarantella et la rendre compatible avec des contraintes instrumentales nouvelles (c'est ainsi qu'établissant des rôles structurels plus strictement répartis, accords de tonique et de dominante confiés à la main gauche de l'accordéon se substituent à ZT 1 et ZT 2). Dans d'autres cas, diverses percussions - tambours, triangle, bouteilles frappées, etc. - se voient confier l'exclusivité de l'alternance binaire/ternaire (les instruments mélodiques sont alors confinés dans une seule dimension métrique). Enfin, l'évolution a pu conduire à la disparition pure et simple de l'articulation binaire/ternaire et à la mise en place de structures 
fixes (le plus souvent ternaires) non variées et non ornées, notamment dans le cas de tarantelle plus modernes et "folklorisées " de Sicile ou de Campanie (cf. disque 3, face A, plage 5 et B, plage 7).

Cette perte de la variation conduit de proche en proche à la négation de notre système. Ce système, comme on l'a vu, procède par agencements d'énoncés formulaires entrant dans des niveaux classificatoires distincts, eux-mêmes liés par des relations de compatibilité ou d'incompatibilité. Ces énoncés sont en nombre limité (deux à quatre par classes et de l'ordre de la quinzaine en tout pour l'exemple que nous avons traité en détail) ; au maximum de l'ordre de la trentaine dans un exemple particulièrement riche (cf. disque 1 , face $B$, plage 6).

La richesse du système et l'impression de renouvellement que laisse paraître la forme sont attribuables non pas au nombre des éléments en présence, mais aux multiples modalités de combinaisons de ces classes constitutives et des formules qu'elles contiennent. Des combinaisons complémentaires sont en outre fournies par le système d'ornementation lui-même qui, tout en étant assujetti étroitement aux systèmes métrique et tonal, apporte des possibilités de micro-variations complémentaires.

La dégradation de la tarantella passe par la réduction du nombre d'embranchements de notre arbre et par l'érosion progressive des variantes s'inscrivant dans les classes terminales. Dans les tarantelle " folklorisées », il ne reste que le tronc et quelques combinaisons figées dans un jeu où la distribution des composantes est fixée une fois pour toutes, ainsi l'exemple - disque 4, face $\mathrm{A}$, plage 8 - joué à l'accordéon et représenté dans la figure 13 :

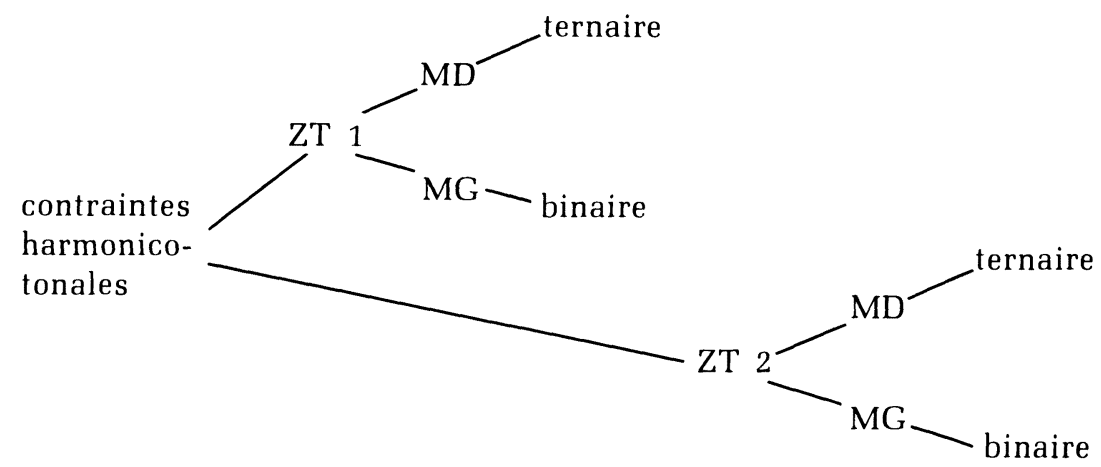


Le point d'aboutissement de cette évolution est l'élimination de l'ornementation et du jeu binaire/ternaire. Seule demeure l'alternance obligée de ZT 1 et ZT 2 prenant valeur de zones de tonique/dominante et configurant étroitement la tarantella dans une forme strophique. Ainsi l'exemple provenant de Capri (disque 3, face A, plage 5), représenté à la figure 14 :

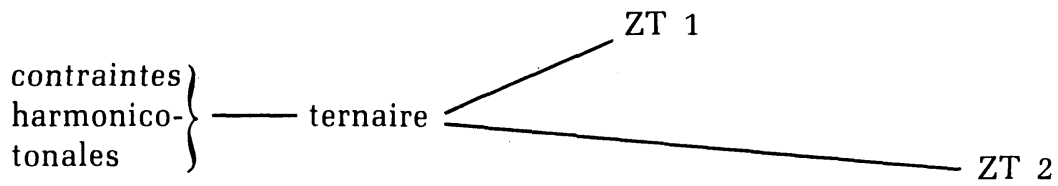

Figure 14

L'arbre perd ainsi ses branches terminales au profit d'une structure unitaire; outre qu'ils sont moins nombreux que dans les formes anciennes, les terminaux se voient désormais sollicités toujours de la même façon et viennent à perdre leur statut de classe constitutive pour prendre la forme de simples occurrences répétées identiquement d'une strophe à l'autre. L'ordre de l'énoncé immuable se substitue à celui de choix formulaires, et dans ces nouvelles règles, la danse perd toute sa vie.

Or, si l'on veut bien solliciter un moment la métaphore, il se trouve que, dans la nature, ce sont les branches et les branchettes qui assurent le renouvellement et la vie même de l'arbre. Aussi, en perdant son foisonnement de ramifications ultimes, l'arbre de la tarantella, dressé à des fins de connaissance par le chercheur, risque de devenir un arbre mort, mangé par le temps, que seule l'émergence d'imaginations nouvelles ou renouvelées pourrait ranimer. 


\section{NOTES}

1. Cet article a été écrit avant que je ne prenne connaissance d'une étude fort détaillée de Giovanni Giuriati (1982) sur la Tarantella de Montemarano en Campanie. Giuriati m'ayant fort aimablement adressé son manuscrit, j'ai fait figurer en note les points qui complètent mon propos.

2. Giannattasio rappelle utilement que «le mot (tarentelle) regroupe plusieurs expressions chorégrapho-musicales : les tarentelles lentes qui accompagnent le chant d'amour en Lucanie, les pizzichepizziche qui proviennent du tarentisme (le pizzico est la morsure de l'araignée) mais qui sont devenues danses d'amour dans les Pouilles, en Calabre, et en Sicile, les différentes tarentelles plus ou moins folklorisées de Campanie » (1979:7-8).

3. J'ai pu ailleurs (1975: 6 et sq.) mentionner les problèmes que rencontrent nécessairement les utilisateurs de cette méthode dès lors qu'ils doivent prendre en compte la multiplicité des composantes musicales susceptibles d'avoir des fonctions structurantes spécifiques.

4. La tarantella de Montemarano, décrite par Giuriati, répond également au principe d'alternance tonale; cette alternance est, selon lui, "commune à presque toutes les musiques de danse d'Italie centre-méridionale » (1982:21).

5. Les modalités d'alternance de ces espaces sont variables selon les styles et les régions. Il convient de noter que (a b) occupe un espace métrique correspondant à deux ou à quatre mesures et que certaines formes combinent ces deux structurations (ainsi l'exemple du disque 1 , face A, plage 5). Analysant les techniques de variations utilisées dans la tarantella de Montemarano, Giovanni Giuriati propose une segmentation opérant sur deux, trois et quatre mesures.

6. Sur ce point, cf. également Giannattasio (1980:12) et Giuriati (1982:21). Ce dernier, évoquant à propos de la tarantella de Montemarano les problèmes d'ambiguïté rythmique, parle d'une "oscillation continue entre $4 / 4$ et $12 / 8$ ". 


\section{RÉFÉRENCES}

CRIVELLI, C.

1979 : "Nota musicologica », notice du disque Calabria 1, strumenti, Fonit Cetra, collection «I Suoni » dirigée par Diego Carpitella, Cetra SU 5001.

GIANNATTASIO, F.

1979 : "Instruments à percussion et rythmes dans les danses d'Italie du sud », projet de thèse de $3^{\mathrm{e}}$ cycle, Université de Paris X Nanterre, ronéoté.

GIANNATTASIO, F. et LORTAT-JACOB, B.

1982 : “Modalita' di improvvisazione nella musica sarda, due modelli », Culture musicali, Quaderni di etnomusicologia,

GIURIATI, G. I/1, 3-35.

1982 : «Un procedimento compositivo caleidoscopico : la tarantella di Montemarano », Culture musicali, Quaderni di etnomusicologia, II/1 : 19-72.

LORTAT-JACOB, B.

1975 : «Quelques problèmes d'analyse musicale », Revue de Musicologie, LXI/1, 3-34.

1981: "Danse de Sardaigne : composition, renouvellement", in "Ethnomusicologie et représentations de la musique ", Études réunies et présentées par Gilbert Rouget, Le Courrier du CNRS, 42-43.

NATTIEZ, J.-J.

1975 : Fondements d'une sémiologie de la musique. Paris, Union générale d'Éditions (10-18: $\left.\mathrm{N}^{\circ} 1017\right)$.

\section{Discographie : exemples cités}

1. Calabria 1, strumenti : Enregistrements et notice de Roberta Tucci, note musicologique et transcriptions de Carlo Crivelli, disque $30 \mathrm{~cm} /$ 33 tours, Fonit Cetra, collection « I Suoni » dirigée par Diego Carpitella, Cetra SU 5001.

2. Calabria bella, Dove t'hai lasciate? : Italian Folk Music collected in New-York, New-Jersey and Rhode Island. Enregistrements et notice de Anna L. Chairetakis, disque $30 \mathrm{~cm} / 33$ tours, Ethnic Folkways FES 34042.

3. Folk Music from Italy : Enregistrements et notice de Walther Henig, disque $30 \mathrm{~cm} / 33$ tours, Ethnic Folkways FE 4520.

4. La zampogna in italia e le launeddas : Enregistrements et notice de Roberto Leydi et Bruno Pianta, disque $30 \mathrm{~cm} / 33$ tours, Albatros VPA 8149. 